\title{
Chinese adaptation of the Ten-Item Internet Gaming Disorder Test and prevalence estimate of Internet gaming disorder among adolescents in Taiwan
}

\author{
YU-CHUAN CHIU ${ }^{1}$, YUAN-CHIEN PAN ${ }^{2}$ and YU-HSUAN LIN $3,4,5,6 *$ \\ ${ }^{1}$ Department of Psychiatry, MacKay Memorial Hospital, Taipei, Taiwan \\ ${ }^{2}$ Department of Psychology, National Taiwan University, Taipei, Taiwan \\ ${ }^{3}$ Institute of Population Health Sciences, National Health Research Institutes, Miaoli, Taiwan \\ ${ }^{4}$ Department of Psychiatry, National Taiwan University Hospital, Taipei, Taiwan \\ ${ }^{5}$ Department of Psychiatry, College of Medicine, National Taiwan University, Taipei, Taiwan \\ ${ }^{6}$ Institute of Health Behaviors and Community Sciences, College of Public Health, National Taiwan University, Taipei, Taiwan
}

(Received: December 28, 2017; revised manuscript received: April 6, 2018; second revised manuscript received: May 28, 2018; third revised manuscript received: August 19, 2018; accepted: August 20, 2018)

\begin{abstract}
Background and aims: Internet gaming disorder (IGD) is an increasingly important topic and has been included in the Diagnostic and Statistical Manual of Mental Disorders (DSM-5) research criteria. This study aims to validate the Chinese version of the Ten-Item Internet Gaming Disorder Test (IGDT-10), a self-reported questionnaire based on DSM-5 IGD criteria, and to estimate the prevalence of IGD in adolescents. Methods: The IGDT-10 was translated to Chinese as a 10-item questionnaire rated on a 3-point Likert scale to evaluate the symptoms of IGD. Overall, 8,110 students from grade four to senior high who played Internet games were administered the questionnaire. In addition, 76 senior high-school students were interviewed using DSM-5 criteria to determine the optimal cut-off point that ensures adequate sensitivity, specificity, and diagnostic accuracy. The cut-off point was determined using the Youden's index and optimal diagnostic accuracy. Results: The Chinese version of the IGDT-10 showed good internal consistency (Cronbach's $\alpha=.85$ ) and adequate diagnostic efficiency (area under the curve $=0.810$ ). Through interviews, the optimal cut-off point was determined to be five out of the nine criteria (Youden's index: $42.1 \%$, diagnostic accuracy: $86.8 \%$, sensitivity: $43.8 \%$, and specificity: $98.3 \%$ ). In this study, the prevalence of IGD among adolescent gamers was $3.1 \%$. Conclusion: Findings evidence the validity and diagnostic accuracy of the IGDT-10 in the assessment of IGD.
\end{abstract}

Keywords: Internet gaming disorder, Internet addiction, gaming addiction

\section{INTRODUCTION}

As Internet gaming has become more prevalent and involved in people's daily lives, the risk of addiction is increasing and may become a major health problem. The International Classification of Diseases, 11th Revision, beta draft designates "Gaming Disorder" as characterized by a pattern of recurrent gaming online or offline manifested by "(a) impaired control over gaming (e.g., onset, frequency, intensity, duration, termination, and context); (b) increasing priority given to gaming to the extent that gaming takes precedence over other life interests and daily activities; and (c) continuation or escalation of gaming despite the occurrence of negative consequences. The behavior pattern is of sufficient severity to result in significant impairment in personal, family, social, educational, occupational, or other important areas of functioning" (World Health Organization, 2018). Griffiths (1996) first described technological addiction as behavioral addiction that involves humanmachine interaction and is non-chemical in nature. Gambling disorder is currently the only behavioral addiction categorized in the "substance-related and addictive disorders" section of the current Diagnostic and Statistical Manual of Mental Disorders (DSM-5); this is due to similarities in symptomatology and biology (Potenza et al., 2003), genetics (Slutske et al., 2000), and treatments (Hodgins, Currie, \& el-Guebaly, 2001; Petry et al., 2006; Petry, Weinstock, Ledgerwood, \& Morasco, 2008). Internet gaming disorder (IGD) is listed in Section 3 (Emerging Measures and Models) of the DSM-5 (American Psychiatric Association, 2013). The APA has identified IGD as a potential disorder that might be included in the future versions of the DSM, and the APA Substance-Related Disorders Work Group has asked for research on IGD, including its prevalence, validity of criteria, and crosscultural reliability (Hasin et al., 2013). Recent prevalence

\footnotetext{
* Corresponding author: Yu-Hsuan Lin, MD, PhD; Institute of Population Health Sciences, National Health Research Institutes, 35 Keyan Road, Zhunan, Miaoli County 35053, Taiwan; Phone: +886 37246166 ext. 36383; Fax: +886 37 586261; E-mail: yuhsuanlin@nhri.org.tw
}

This is an open-access article distributed under the terms of the Creative Commons Attribution-NonCommercial 4.0 International License, which permits unrestricted use, distribution, and reproduction in any medium for non-commercial purposes, provided the original author and source are credited, a link to the CC License is provided, and changes - if any - are indicated. 
research into IGD has shown cross-cultural variability (Przybylski, Weinstein, \& Murayama, 2017) with East Asian cultures having higher IGD prevalence than Western cultures; however, the prevalence rate in these Eastern culture studies is very likely to be inflated (Kuss, Griffiths, Karila, \& Billieux, 2014). While one large-scale crosscultural prevalence study has examined IGD (Przybylski et al., 2017), no large-scale IGD study based on DSM-5 criteria has been conducted in any Asian countries.

Although several validated self-reported questionnaires regarding IGD exist (Lemmens, Valkenburg, \& Gentile, 2015; Petry et al., 2014; Pontes \& Griffiths, 2015; Rehbein, Kliem, Baier, Mößle, \& Petry, 2015; Vadlin, Åslund, \& Nilsson, 2015), few questionnaires have been specifically evaluated for individual DSM-5 criteria of IGD, and none have been created in Mandarin or studied in an Asian country. There exists a need for a questionnaire where each item corresponds to the appropriate DSM-5 nine-item criteria of IGD, enabling research into the clinical significance of each DSM-5 item and its relation to diagnostic accuracy, sensitivity, and specificity.

The diagnostic stability of IGD is uncertain due to a lack of longitudinal and cross-cultural studies. Increasingly younger people are becoming Internet gamers, but most studies have included adult groups (Mihara \& Higuchi, 2017). For example, the participants in a recent large-scale cross-country study in Germany, England, and the United States were older than 18 years (Przybylski et al., 2017). To examine diagnostic stability and ensure early prevention, a detailed survey of elementary-, junior high-, and highschool students is thus required.

This study adopted the Ten-Item Internet Gaming Disorder Test (IGDT-10), a DSM-5 criteria-based questionnaire, and validated it through structured interviews in the Mandarin language, estimated the prevalence of IGD in adolescents, and examined the estimated prevalence of IGD in different age stratifications among adolescents.

\section{METHODS}

\section{Participants and procedure}

The participants were 8,110 students $(5,133$ boys and 2,977 girls) recruited from 169 schools (112 elementary, 39 junior high, and 18 senior high schools) in Taiwan during August 2016-June 2017. Their ages ranged from 10 to 18 years $(M=13.17, S D=1.79)$. All participants played at least one computer-based online game. After submitting informed consent, all participants completed a written survey that collected data on participants' demographics, IGD symptoms, and Internet gaming characteristics. Initially, 113 senior highschool students were invited to participate in the interview sessions as they self-reported playing mobile-based or computer-based online games; 76 of these students reported playing computer-based online games and were thus included in this study. The structured interviews were conducted individually by well-trained interviewers (senior graduate students who majored in clinical psychology) to validate and determine the cut-off point of IGDT-10. The interviewers completed a standardized two-session training workshop ( $3 \mathrm{hr} /$ session) for the structured interview designed for evaluating the IGD diagnosis in the DSM-5. Two experienced psychiatrists who were experts in the field of Internet addiction taught and supervised the training workshop. During the training sessions, all trainees were required to finish at least two mock interviews, and their performance was discussed in detail to ensure that the interviews are interrater reliable. All mock interviews were videotaped. The interview session lasted on average for 20-30 $\mathrm{min}$. The IGD structured interview was based on the research criteria of IGD in DSM-5.

\section{Measures}

Ten-Item Internet Gaming Disorder Test (IGDT-10). The IGDT-10 is a self-reported scale for assessing IGD. The instrument was developed based on the DSM-5 nine-item criteria of IGD. Each criterion was operationalized using a single item, except for the last criterion referring to "jeopardy or losing a significant relationship, job, or educational or career opportunity because of participation in Internet games," which was operationalized using two items given its complexity and description of more than one construct. Participants were asked to rate items on a 3-point Likert scale labeled 0 (never), 1 (sometimes), and 2 (often). The IGDT-10 items were recoded into a "yes" (1) or "no" (0) format to resemble the dichotomous structure of IGD in the DSM-5. Because the DSM-5 criteria of IGD suggest that behaviors are frequently repeated or continuously present, only "often" answers were recoded as "yes." Given that questions 9 and 10 are related to the same criterion, they were combined in the scoring, that is, answering "often" on both Item 9 or 10 (or both items) scores only 1 point. Thus, the composite score of IGDT-10 ranged from 0 to 9 , with higher scores indicating more IGD symptomatology.

The Chinese version of IGDT-10 was developed in this study. Double back-translation was performed to avoid language bias. First, the IGDT-10 items were translated into Chinese by Dr. Y-HL, a board-certified psychiatrist experienced in substance-related disorder and Internet addiction. The initial results were then reviewed by another bilingual psychiatrist, Dr. Y-CC for back-translation. Subsequently, all authors carefully reviewed the initial backtranslation and compared it with the original IGDT-10. The original and Chinese versions of IGDT-10 are listed in Table 1. Orsolya Király, the corresponding author of the original IGDT-10 (Király, Sleczka, et al., 2017), was also invited to review our translation process.

Cronbach's $\alpha$ s for IGDT-10 were .68 and .79, respectively, based on the dichotomized nine items (Király, Sleczka, et al., 2017) and the original 10 items with three response options (Király, Toth, Urban, Demetrovics, \& Maraz, 2017). Cronbach's $\alpha$ for the Chinese version of IGDT-10 was .85 , based on the original 10 items with three response options. The cut-off point of the IGDT-10 Chinese version was determined through receiver-operating characteristic (ROC) analysis, with clinical interview as the standard.

DSM-5 diagnostic criteria of IGD. The DSM-5 diagnostic criteria of IGD comprise nine items: preoccupation, withdrawal, tolerance, loss of control, abandoning other activities, continued excessive use despite psychosocial problems, deceiving regarding online gaming, escape, and 
Table 1. The original and the Chinese version of IGDT-10

The original IGDT-10

The Chinese version of IGDT-10

Please read the statements below regarding online gaming. The questionnaire refers to ONLINE GAMES, but the reference to "game" or "gaming" is used for the sake of simplicity. Please, indicate on the scale from 0 to 2 (never, sometimes, and often) to what extent and how often these statements applied to you over the PAST 12 MONTHS!

1. When you were not playing, how often have you fantasized about gaming, thought of previous gaming sessions, and/or anticipated the next game?

2. How often have you felt restless, irritable, anxious and/or sad when you were unable to play or played less than usual?

3. Have you ever in the past 12 months felt the need to play more often or played for longer periods to feel that you have played enough?

4. Have you ever in the past 12 months unsuccessfully tried to reduce the time spent on gaming?

5. Have you ever in the past 12 months played games rather than meet your friends or participate in hobbies and pastimes that you used to enjoy before?

6. Have you played a lot despite negative consequences (for instance, losing sleep, not being able to do well in school or work, having arguments with your family or friends, and/or neglecting important duties)?

7. Have you tried to keep your family, friends, or other important people from knowing how much you were gaming or have you lied to them regarding your gaming?

8. Have you played to relieve a negative mood (for instance, helplessness, guilt, or anxiety)?

9. Have you risked or lost a significant relationship because of gaming?

10. Have you ever in the past 12 months jeopardized your school or work performance because of gaming?
請閱讀以下關於線上遊戲的敘述。這份問卷有些簡化成 「遊戲」或「玩遊戲」的字句，指的就是線上遊戲。請 依照你過去12個月的情形和頻率，在每個敘述選擇0分到 2分 (從來沒有、有時候、經常) 的評分。

當你沒有玩線上遊戲時，你多常幻想自己在玩線上遊戲、 想著前幾次玩遊戲的事; 或期待下一次的遊戲?

當你不能玩線上遊戲或是玩得比平常少的時候, 你多常感 到靜不下心、煩躁、焦慮、或悲傷?

在過去的12個月裡, 你感覺需要更常玩線上遊戲, 或打更 久的時間才覺得你玩夠了?

\section{在過去的12個月裡，你曾經試著減少花在線上遊戲的時}

間，但沒有成功？

在過去的12個月裡，你曾經會玩線上遊戲而沒和朋友見 面, 或不再從事你以前常參加的嗜好活動?

即使線上遊戲的負面影響（例如減少睡眠、無法把學業或 工作做好、與家人或朋友爭吵、或無視於重要的責任), 你還是玩很多?

你曾試著不讓你的家人、朋友或其他重要的人知道你玩線 上遊戲的時間, 或你曾對他們謊稱你玩線上遊戲的情 形?

你曾玩線上遊戲來舒解負面的情緒（例如感到無助、內 疚、或焦慮)?

你曾因為玩線上遊戲而可能危害或失去重要的人際關係?

在過去的12個月裡，你曾經因為玩線上遊戲而使你在學校 或工作的表現陷入重大危機?

Note. IGDT-10: Ten-Item Internet Gaming Disorder Test.

negative consequences. We developed a structured interview schedule to examine the DSM- 5 criteria of IGD based on two recent international recommendations (Griffiths et al., 2016; Petry et al., 2014). The diagnosis for IGD in the structured interview was based on the cut-off value of 5 suggested in the DSM-5.

Relevant features of IGD. To assess the relevant features of Internet gaming, all the participants reported whether they ever spent money on online games, the average time (hours per week) spent on Internet gaming during weekdays and weekends, body mass index (BMI), and myopia $(0=$ normal, $1=$ less than -3.0 dioptres, $2=-3.0$ to -6.0 dioptres, $3=$ more than -6.0 dioptres).

\section{Statistical analysis}

The diagnostic ability of the IGDT-10 for IGD was evaluated through a ROC analysis. The area under the ROC curve (AUC) is a measure of the diagnostic efficacy of IGDT-10. The sensitivity, specificity, Youden's index, and diagnostic accuracy of IGDT-10 were evaluated for the diagnostic positive and negative groups. Sensitivity and specificity indicate the true positive and negative rates, respectively. Youden's index is defined as sensitivity + specificity -1 .
In addition, positive (negative) predictive rate measures the proportion of diagnostic positives among participants with IGDT-10 scores higher (lower) than the cut-off points. Diagnostic accuracy, which indicates the percentage of all correct decisions, is obtained by dividing the number of true positives and negatives by the number of all decisions.

The cut-off point of IGDT-10 is optimal for diagnosis when the score is accompanied by the highest diagnostic accuracy and the optimal Youden's index. To confirm the validity of the IGDT-10 cut-off score proposed in this study and to explore the characteristics of Internet gaming, participants were further classified into IGD and non-IGD groups according to the cut-off point of the IGDT-10. The demographic data and relevant characteristics of mobile gaming were further compared between these two groups using a $\chi^{2}$ test or $t$-test. $p<.05$ was considered statistically significant. The analyses were conducted using SPSS 18.0 for Windows (SPSS, Chicago, IL, USA).

\section{Ethics}

Informed consent was obtained from all participants and their parents prior to assessment for those younger than 18 years of age. This study was approved by the institutional 
Table 2. Sensitivity, specificity, PPR, NPR, DA, and Youden's index of cut-off points in IGDT-10 between diagnostic positive and negative groups $(N=76)$

\begin{tabular}{lcccccc}
\hline Cut-off point & Sensitivity (\%) & Specificity (\%) & PPR (\%) & NPR (\%) & DA (\%) & Youden's index (\%) \\
\hline 1 & 81.3 & 63.3 & 37.1 & 92.7 & 67.1 & 44.6 \\
2 & 68.8 & 81.7 & 50.0 & 90.7 & 78.9 & 50.4 \\
3 & 56.3 & 88.3 & 56.3 & 88.3 & 81.6 & 44.6 \\
4 & 56.3 & 91.7 & 64.3 & 88.7 & 84.2 & 47.9 \\
5 & 43.8 & 98.3 & 87.5 & 86.8 & 86.8 & 42.1 \\
6 & 18.8 & 100.0 & 100.0 & 82.2 & 82.9 & 18.8 \\
7 & 18.8 & 100.0 & 100.0 & 82.2 & 82.9 & 18.8 \\
8 & 12.5 & 100.0 & 100.0 & 81.1 & 81.6 & 12.5 \\
\hline
\end{tabular}

Note. IGDT-10: Ten-Item Internet Gaming Disorder Test; Sensitivity: the proportion of true positive rates; Specificity: the proportion of the true negative rates; Youden's index: defined as sensitivity + specificity - 1; PPR: positive predictive rate, indicated the proportion of diagnostic positives among participants with IGDT-10 scores greater than cut-off points; NPR: negative predictive rate, indicated the proportion of diagnostic negatives among participants with IGDT-10 scores less than cut-off points; DA: diagnostic accuracy, indicated the percentage of all correct decisions, which was the result of dividing the number of true positives and true negatives by the number of all decisions. $\mathrm{AUC}=0.810$.

review board of National Taiwan University Hospital and carried out in accordance with the latest version of the Declaration of Helsinki.

\section{RESULTS}

\section{Determination of cut-off point}

To determine the cut-off score of IGDT-10, structured interviews based on the DSM-5 diagnostic criteria for IGD were used as the gold standard. The participants invited for the structured interview process were 76 senior high-school students ( 64 boys and 12 girls), all of whom belong to one mixed-gender private high school in northern Taiwan. The institution ensured that their students were available for the interview; the institution had previously collaborated with our research team. The age of the students ranged from 15 to 16 years $(M=15.66, S D=0.47)$. Participants who reported having played at least one computer-based online game completed the interview (age: $M=15.61$, $S D=0.49)$. Their composite scores on the IGDT-10 ranged from 0 to $8(M=1.36, S D=2.04)$. Eight of the 76 students were diagnosed as having IGD. Table 2 presents the ROC analysis profile for IGDT-10 between the diagnostic positive and negative groups. The AUC was 0.810 , evidencing the high diagnostic efficiency of IGDT-10. A cut-off point of 4/5 was optimal for discriminating cases of IGD from diagnostic negatives (Youden's index: 42.1\%, diagnostic accuracy: $86.8 \%$, sensitivity: $43.8 \%$, and specificity: $98.3 \%)$

\section{IGD prevalence estimate and criteria endorsement}

Figure 1 shows the frequency distribution of the number of IGD symptoms reported in the total sample; $71.5 \%$ online gamers did not report any IGD symptoms. Based on the aforementioned cut-off point of 5 for classifying disordered gamers, IGD prevalence estimate in the present sample was $3.1 \%$.

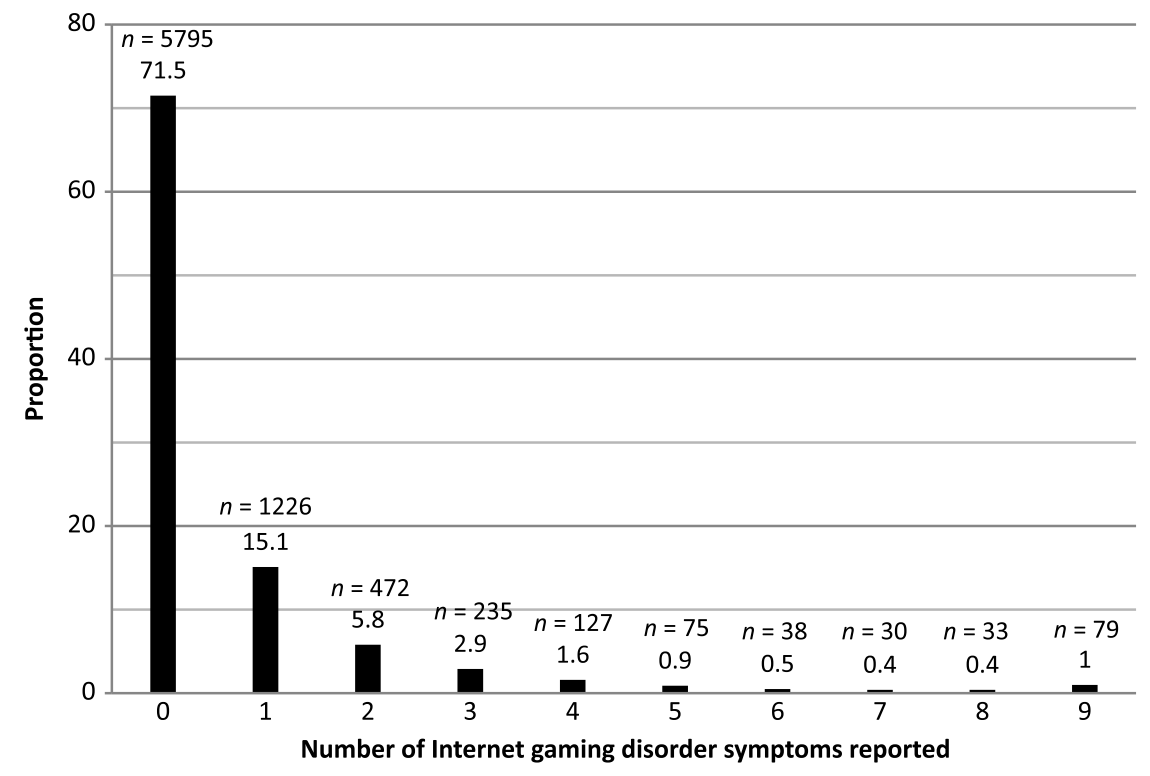

Figure 1. Frequency distribution of the number of Internet gaming disorder symptoms in the total sample $(N=8,110)$ 
Table 3. Sensitivity, specificity, and endorsement of nine criteria IGD in the IGD group and the total sample

\begin{tabular}{|c|c|c|c|c|c|c|}
\hline \multirow[b]{2}{*}{ Criteria } & \multirow[b]{2}{*}{ Sensitivity (\%) } & \multirow[b]{2}{*}{ Specificity (\%) } & \multicolumn{2}{|c|}{$\begin{array}{l}\text { Endorsement among the IGD } \\
\text { group }(N=255)\end{array}$} & \multicolumn{2}{|c|}{$\begin{array}{l}\text { Endorsement in the total sample } \\
\qquad(N=8,110)\end{array}$} \\
\hline & & & $n$ & $\%$ & $n$ & $\%$ \\
\hline 1. Preoccupation & 83.9 & 92.2 & 214 & 83.9 & 830 & 10.2 \\
\hline 2. Withdrawal & 80.4 & 96.6 & 205 & 80.4 & 473 & 5.8 \\
\hline 3. Tolerance & 85.1 & 94.7 & 217 & 85.1 & 636 & 7.8 \\
\hline 4. Loss of control & 71.0 & 93.5 & 181 & 71.0 & 693 & 8.5 \\
\hline 5. Give up other activities & 74.1 & 98.0 & 189 & 74.1 & 350 & 4.3 \\
\hline 6. Continuation & 83.9 & 96.0 & 214 & 83.9 & 529 & 6.5 \\
\hline 7. Deception & 70.2 & 98.2 & 179 & 70.2 & 318 & 3.9 \\
\hline 8. Escape & 84.7 & 90.1 & 216 & 84.7 & 995 & 12.3 \\
\hline 9. Negative consequences & 67.8 & 97.8 & 173 & 67.8 & 347 & 4.3 \\
\hline
\end{tabular}

Note. SD: standard deviation; IGD: Internet gaming disorder; IGDT-10: Ten-Item Internet Gaming Disorder Test; BMI: body mass index; OR: odds ratio; $d$ : Cohen's $d$.

Table 3 shows the endorsement of each IGD criterion (i.e., sensitivity and specificity) in the total sample and among the IGD group. Sensitivity ranged from $67.8 \%$ (negative consequences) to $85.1 \%$ (tolerance). Specificity ranged from $90.1 \%$ (escape) to $98.2 \%$ (deception). In the IGD group, the most common IGD symptom was tolerance (85.1\%), followed by escape (84.7\%), continuation ( $83.9 \%)$, preoccupation $(83.9 \%)$, and withdrawal $(80.4 \%)$. In the total sample, the most common IGD symptoms were escape $(12.3 \%)$, preoccupation $(10.2 \%)$, and loss of control (8.5\%).

\section{Demographic data and relevant characteristics of IGD}

All participants were divided into three subsamples based on their level of education (fourth to sixth grade, junior high, and senior high school). Each subsample was further classified into IGD and non-IGD groups according to the cut-off point of 5 in the IGDT-10. Overall, the proportion of IGD was $3.8 \%$ ( $82.5 \%$ boys) among elementary-school students, $2.9 \%$ among junior high-school students ( $83.2 \%$ boys), and $1.9 \%$ among senior high-school students (91.3\% boys). Among elementary-, junior high-, and senior high-school students, $5.3 \%, 3.6 \%$, and $2.6 \%$ of boys and $1.7 \%, 1.5 \%$, and $0.4 \%$ of girls, respectively, were diagnosed as showing symptoms of IGD.

Table 4 presents the group comparisons of demographic data and relevant characteristics of IGD. Significant gender differences exist between the IGD and non-IGD groups in elementary school and junior and senior high-school students. Compared with the non-IGD group, the IGD group exhibited significantly higher proportions of money and time spent on Internet gaming per week. Furthermore, significant BMI differences were noted between the IGD and the non-IGD groups in elementary school students but not in junior high and senior high-school students. However, the proportion of myopia did not differ significantly between the IGD and non-IGD groups in any group.

\section{DISCUSSION}

Several findings in this study are of significance. The cut-off point of 5/9 is consistent with the DSM- 5 diagnostic criteria, which requires five of nine criteria to be met for diagnosis. Our cut-off point of $5 / 9$ is also consistent with research on IGD and IGDT-10 (Király, Sleczka, et al., 2017). However, the original IGDT-10 cut-off point was determined using latent class analysis and not structured interviews performed by qualified interviewers. Our cut-off point of $5 / 9$ is also consistent with a validation study of the 5/9 cut-off point of the DSM-5 criteria using interviews (Ko et al., 2014), but no large-scale sampling was performed.

Regarding the prevalence estimate of IGD, this study shows some level of consistency with earlier studies. Our results reveal a significant gender difference in IGD. Male students in this study were more likely to meet the IGD criteria, consistent with other culture studies (Haagsma, Pieterse, \& Peters, 2012; Rehbein et al., 2015; Strittmatter et al., 2015). The prevalence estimate rate in this study was $3.1 \%$, and approximately $71.5 \%$ of students failed to meet any IGD criteria. This result is consistent with past research that has demonstrated that a large proportion of Internet gaming users are not pathological users (Király, Sleczka, et al., 2017), and that around two thirds of Internet gamers show no symptoms of misuse (Przybylski et al., 2017). However, our prevalence of $3.1 \%$ is higher compared with that in Europe (1.16\% in Germany; Rehbein et al., 2015 and $2.7 \%$ in the UK; Przybylski et al., 2017) and North America $(1.2 \%-2.8 \%$ in the United States; Przybylski et al., 2017). Studies have shown that Asian populations may have higher IGD rates, with rates as high as $9.9 \%$ in Singapore (Gentile et al., 2011), $15.7 \%$ in Hong Kong (Wang et al., 2014), $4.8 \%-5.8 \%$ in China (Yu, Li, \& Zhang, 2015), and $10.8 \%$ in Korea (Koo, Han, Park, \& Kwon, 2017). However, the measurement used in these Asian studies, with the exception of Korean study (Koo et al., 2017), does not directly correspond to the DSM-5 criteria. One reason for the high prevalence estimate rates is the non-random sampling methods applied in these studies. Screening methods and instruments may also inherently overestimate prevalence rates when the disorder (e.g., IGD) has low prevalence in the population (Maraz, Kiraly, \& Demetrovics, 2015).

Compared with other populations, characteristics of symptomatology frequency with regard to IGD in this study had some similarities and some differences. Among all Internet gamers, the most frequently met criteria were 


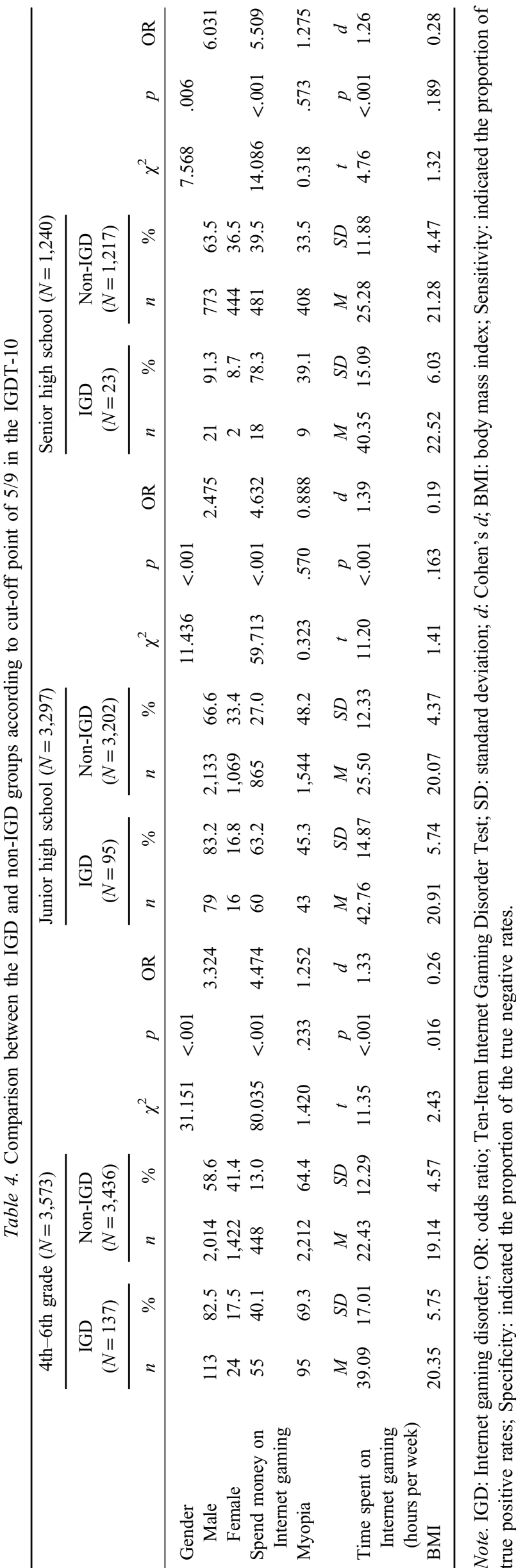

escape (12.3\%), preoccupation (10.3\%), and loss of control $(8.5 \%)$. In the original IGDT-10 study, the most commonly met symptoms were preoccupation $(20.1 \%)$, continuation $(15.5 \%)$, and escape $(13.0 \%)$ in a Hungarian sample population aged 14-64 years (Király, Sleczka, et al., 2017). Escape $(5.30 \%)$ and preoccupation $(3.91 \%)$ were also the most common symptoms noted in a German population aged 13-18 years, which is close to this study's demographic (Rehbein et al., 2015). Among those who met the diagnostic criteria for IGD, the three most common symptoms were tolerance $(85.1 \%)$, escape $(84.7 \%)$, and continuation or preoccupation (both at $83.9 \%$ ). In the original IGDT-10 study, the most common symptoms among participants meeting the IGD diagnosis were continuation $(92.8 \%)$, negative consequences $(81.2 \%)$, and preoccupation $(76.8 \%)$. Escape and preoccupation overlapped among all three studies as commonly met criteria among all users.

This study indicates that the elementary group has the highest prevalence estimate rate of IGD, and IGD prevalence estimate rate decreases as the students' age increases. While the gaming time increased with the students' age, the prevalence estimate rate of IGD decreased. One explanation might be that the association between gaming time and IGD is usually weak and that gaming time is a poor predictor of IGD (Király, Sleczka, et al., 2017). No other study has investigated IGD in adolescents as young as 10 years old (fourth grade students). One explanation for this age difference is that IGD may not be a permanent phenomenon (Konkolÿ Thege, Woodin, Hodgins, \& Williams, 2015; Rothmund, Klimmt, \& Gollwitzer, 2016). Another cause might be that the impulse-control capabilities of elementaryschool students are not yet mature. Adolescents are particularly at risk for addiction (Volkow, Koob, \& McLellan, 2016). The prefrontal cortex and other cortical networks that are critical for judgement and self-regulation do not fully mature until the age of 21-25 years (Giedd et al., 1999). Compared with female students, male students in this study were more likely to meet the IGD criteria, consistent with the results in other cultures (Haagsma et al., 2012; Rehbein et al., 2015; Strittmatter et al., 2015). Gender differences in IGD may involve neurobiological changes. Among the proposed mechanisms are the altered hippocampal volume and functional connectivity (Yoon et al., 2017); altered plasma metabolites, such as arabitol, myo-inositol, methionine, pyrrole-2-carboxylic acid, and aspartic acid (Cho et al., 2017); and stress vulnerability correlated with cortisol response (Kaess et al., 2017).

No statistical differences in myopia were noted between IGD and non-IGD students in all age groups, and only elementary-school students displayed a difference in BMI between IGD and non-IGD students. Longer follow-up periods than usual are required to observe a significant difference between IGD and non-IGD groups with regard to myopia. Students with IGD spent more money on games compared with those without IGD in all three age groups. The potential financial impact of IGD upon adolescents should be further investigated.

Several study limitations should be noted when interpreting our findings. First, the sample was restricted to adolescent students in Taiwan, which limits the generalization of our 
findings. Second, IGD was solely assessed on the basis of the participants' self-reported questionnaires and responses to the diagnostic interview. Additional supplementary information may confirm these symptoms, including interviews with guardians and other corroborating information. Our interview sample was not randomly selected and was from one single educational institution, which may influence the prevalence estimate rates. The interviews were diagnosed based on the cut-off value of 5, as suggested in the DSM-5. This result might have influenced the determination of the optimal cutoff value in the analysis. Finally, the cross-sectional design of this study limited the possibility of making casual inferences about the relationship between IGD and its health outcomes, such as BMI and myopia.

\section{CONCLUSIONS}

The Chinese version of IGDT-10 is a validated questionnaire based on the DSM-5 criteria for IGD evaluation. A cut-off point of 5/9 determined using structured interviews is consistent with earlier research on IGD criteria. A large sample survey among adolescents indicated a prevalence estimate rate of $3.1 \%$ among Taiwanese students from fourth grade to senior high, with boys being significantly more likely to develop IGD than girls.

Funding sources: This study was supported by the National Taiwan University Hospital (NTUH; grant number: 106N3705). In addition, this study was supported by a grant from National Health Research Institutes of Taiwan (06A1PHPP30-014).

Authors' contribution: Y-CC: contributed in designing the study, revising the article critically for important intellectual content, and final approval of the version to be published. Y-CP: contributed in conceptualizing and designing the study, analysis and interpretation of data, drafting the article, and final approval of the version to be published. Y-HL: contributed in conceptualizing and designing the study, interpretation of data, drafting the article, and final approval of the version to be published.

Conflict of interest: The authors declare no conflict of interest.

Acknowledgements: The authors would like to thank Taipei Computer Association ICT Industry Policy \& Law Center, Ms. Fu-Hua Yu, Ms. Zhi-Ni Zheng, Ms. Yu-Chun Liu, Mr. I-Feng Huang, and Mr. Bo-Yu Wong's excellent technical assistance.

\section{REFERENCES}

American Psychiatric Association. (2013). Diagnostic and statistical manual of mental disorders (5th ed.). Washinton, DC: American Pschiatric Association.
Cho, Y. U., Lee, D., Lee, J.-E., Kim, K. H., Lee, D. Y., \& Jung, Y.-C. (2017). Exploratory metabolomics of biomarker identification for the Internet gaming disorder in young Korean males. Journal of Chromatography B, 1057, 24-31. doi:10.1016/j.jchromb.2017.04.046

Gentile, D. A., Choo, H., Liau, A., Sim, T., Li, D., Fung, D., \& Khoo, A. (2011). Pathological video game use among youths: A two-year longitudinal study. Pediatrics, 127(2), e319-e329. doi:10.1542/peds.2010-1353

Giedd, J. N., Blumenthal, J., Jeffries, N. O., Castellanos, F. X., Liu, H., Zijdenbos, A., Paus, T., Evans, A. C., \& Rapoport, J. L. (1999). Brain development during childhood and adolescence: A longitudinal MRI study. Nature Neuroscience, 2(10), 861863. doi:10.1038/13158

Griffiths, M. D. (1996). Gambling on the Internet: A brief note. Journal of Gambling Studies, 12(4), 471-473. doi:10.1007/ BF01539190

Griffiths, M. D., van Rooij, A. J., Kardefelt-Winther, D., Starcevic, V., Kiraly, O., Pallesen, S., Müller, K., Dreier, M., Carras, M., Prause, N., King, D. L., Aboujaoude, E., Kuss, D. J., Pontes, H. M., Lopez Fernandez, O., Nagygyorgy, K., Achab, S., Billieux, J., Quandt, T., Carbonell, X., Ferguson, C. J., Hoff, R. A., Derevensky, J., Haagsma, M. C., Delfabbro, P., Coulson, M., Hussain, Z., \& Demetrovics, Z. (2016). Working towards an international consensus on criteria for assessing Internet gaming disorder: A critical commentary on Petry et al. (2014). Addiction, 111(1), 167-175. doi:10.1111/add.13057

Haagsma, M. C., Pieterse, M. E., \& Peters, O. (2012). The prevalence of problematic video gamers in the Netherlands. Cyberpsychology, Behavior, and Social Networking, 15(3), 162-168. doi:10.1089/cyber.2011.0248

Hasin, D. S., O'Brien, C. P., Auriacombe, M., Borges, G., Bucholz, K., Budney, A., Compton, W. M., Crowley, T., Ling, W., Petry, N. M., Schuckit, M., \& Grant, B. F. (2013). DSM-5 criteria for substance use disorders: Recommendations and rationale. American Journal of Psychiatry, 170(8), 834-851. doi:10.1176/appi.ajp.2013.12060782

Hodgins, D. C., Currie, S. R., \& el-Guebaly, N. (2001). Motivational enhancement and self-help treatments for problem gambling. Journal of Consulting and Clinical Psychology, 69(1), 50-57. doi:10.1037/0022-006X.69.1.50

Kaess, M., Parzer, P., Mehl, L., Weil, L., Strittmatter, E., Resch, F., \& Koenig, J. (2017). Stress vulnerability in male youth with Internet gaming disorder. Psychoneuroendocrinology, 77, 244-251. doi:10.1016/j.psyneuen.2017.01.008

Király, O., Sleczka, P., Pontes, H. M., Urban, R., Griffiths, M. D., \& Demetrovics, Z. (2017). Validation of the Ten-Item Internet Gaming Disorder Test (IGDT-10) and evaluation of the nine DSM-5 Internet gaming disorder criteria. Addictive Behaviors, 64, 253-260. doi:10.1016/j.addbeh.2015.11.005

Király, O., Toth, D., Urban, R., Demetrovics, Z., \& Maraz, A. (2017). Intense video gaming is not essentially problematic. Psychology of Addictive Behaviors, 31(7), 807-817. doi:10.1037/adb0000316

Ko, C. H., Yen, J. Y., Chen, S. H., Wang, P. W., Chen, C. S., \& Yen, C. F. (2014). Evaluation of the diagnostic criteria of Internet gaming disorder in the DSM-5 among young adults in Taiwan. Journal of Psychiatric Research, 53, 103-110. doi:10.1016/j.jpsychires.2014.02.008

Konkolÿ Thege, B., Woodin, E. M., Hodgins, D. C., \& Williams, R. J. (2015). Natural course of behavioral addictions: A 5-year 
longitudinal study. BMC Psychiatry, 15(1), 4. doi:10.1186/ s12888-015-0383-3

Koo, H. J., Han, D. H., Park, S.-Y., \& Kwon, J.-H. (2017). The Structured Clinical Interview for DSM-5 Internet gaming disorder: Development and validation for diagnosing IGD in adolescents. Psychiatry Investigation, 14(1), 21-29. doi:10.4306/pi.2017.14.1.21

Kuss, D. J., Griffiths, M. D., Karila, L., \& Billieux, J. (2014). Internet addiction: A systematic review of epidemiological research for the last decade. Current Pharmaceutical Design, 20(25), 4026-4052. doi:10.2174/13816128113199990617

Lemmens, J. S., Valkenburg, P. M., \& Gentile, D. A. (2015). The Internet Gaming Disorder Scale. Psychological Assessment, 27(2), 567-582. doi:10.1037/pas0000062

Maraz, A., Kiraly, O., \& Demetrovics, Z. (2015). Commentary on: Are we overpathologizing everyday life? A tenable blueprint for behavioral addiction research. The diagnostic pitfalls of surveys: If you score positive on a test of addiction, you still have a good chance not to be addicted. Journal of Behavioral Addictions, 4(3), 151-154. doi:10.1556/2006.4.2015.026

Mihara, S., \& Higuchi, S. (2017). Cross-sectional and longitudinal epidemiological studies of Internet gaming disorder: A systematic review of the literature. Psychiatry and Clinical Neurosciences, 71(7), 425-444. doi:10.1111/pcn.12532

Petry, N. M., Ammerman, Y., Bohl, J., Doersch, A., Gay, H., Kadden, R., Molina, C., \& Steinberg, K. (2006). Cognitivebehavioral therapy for pathological gamblers. Journal of Consulting and Clinical Psychology, 74(3), 555-567. doi:10.1037/0022-006X.74.3.555

Petry, N. M., Rehbein, F., Gentile, D. A., Lemmens, J. S., Rumpf, H. J., Mossle, T., Bischof, G., Tao, R., Fung, D. S., Borges, G., Auriacombe, M., González Ibáñez, A., Tam, P., \& O’Brien, C. P. (2014). An international consensus for assessing Internet gaming disorder using the new DSM-5 approach. Addiction, 109(9), 1399-1406. doi:10.1111/add.12457

Petry, N. M., Weinstock, J., Ledgerwood, D. M., \& Morasco, B. (2008). A randomized trial of brief interventions for problem and pathological gamblers. Journal of Consulting and Clinical Psychology, 76(2), 318-328. doi:10.1037/0022006X.76.2.318

Pontes, H. M., \& Griffiths, M. D. (2015). Measuring DSM-5 Internet gaming disorder: Development and validation of a Short Psychometric Scale. Computers in Human Behavior, 45 137-143. doi:10.1016/j.chb.2014.12.006

Potenza, M. N., Leung, H. C., Blumberg, H. P., Peterson, B. S., Fulbright, R. K., Lacadie, C. M., Skudlarski, P., \& Gore, J. C. (2003). An FMRI Stroop task study of ventromedial prefrontal cortical function in pathological gamblers. American Journal of Psychiatry, 160(11), 1990-1994. doi:10.1176/appi.ajp.160. 11.1990

Przybylski, A. K., Weinstein, N., \& Murayama, K. (2017). Internet gaming disorder: Investigating the clinical relevance of a new phenomenon. American Journal of Psychiatry, 174(3), 230-236. doi:10.1176/appi.ajp.2016.16020224

Rehbein, F., Kliem, S., Baier, D., Mößle, T., \& Petry, N. M. (2015). Prevalence of Internet gaming disorder in German adolescents: Diagnostic contribution of the nine DSM- 5 criteria in a state-wide representative sample. Addiction, 110(5), 842-851. doi:10.1111/add.12849

Rothmund, T., Klimmt, C., \& Gollwitzer, M. (2016). Low temporal stability of excessive video game use in German adolescents. Journal of Media Psychology, 30(2), 53-65. doi:10.1027/1864-1105/a000177

Slutske, W. S., Eisen, S., True, W. R., Lyons, M. J., Goldberg, J., \& Tsuang, M. (2000). Common genetic vulnerability for pathological gambling and alcohol dependence in men. Archives of General Psychiatry, 57(7), 666-673. doi:10.1001/archpsyc. 57.7.666

Strittmatter, E., Kaess, M., Parzer, P., Fischer, G., Carli, V., Hoven, C. W., Wasserman, C., Sarchiapone, M., Durkee, T., Apter, A., Bobes, J., Brunner, R., Cosman, D., Sisask, M., Värnik, P., \& Wasserman, D. (2015). Pathological Internet use among adolescents: Comparing gamers and non-gamers. Psychiatry Research, 228(1), 128-135. doi:10.1016/j.psychres.2015. 04.029

Vadlin, S., Åslund, C., \& Nilsson, K. W. (2015). Development and content validity of a screening instrument for gaming addiction in adolescents: The Gaming Addiction Identification Test (GAIT). Scandinavian Journal of Psychology, 56(4), 458-466. doi:10.1111/sjop.12196

Volkow, N. D., Koob, G. F., \& McLellan, A. T. (2016). Neurobiologic advances from the brain disease model of addiction. The New England Journal of Medicine, 374(4), 363-371. doi:10.1056/NEJMra1511480

Wang, C.-W., Chan, C. L. W., Mak, K.-K., Ho, S.-Y., Wong, P. W. C., \& Ho, R. T. H. (2014). Prevalence and correlates of video and Internet gaming addiction among Hong Kong adolescents: A pilot study. The Scientific World Journal, 2014, 9. doi: $10.1155 / 2014 / 874648$

World Health Organization. (2018). The ICD-11 classification of mental and behavioural disorders: Clinical descriptions and diagnostic guidelines. Geneva: World Health Organization.

Yoon, E. J., Choi, J.-S., Kim, H., Sohn, B. K., Jung, H. Y., Lee, J.-Y., Kim, D. J., Park, S. W., \& Kim, Y. K. (2017). Altered hippocampal volume and functional connectivity in males with Internet gaming disorder comparing to those with alcohol use disorder. Scientific Reports, 7(1), 5744. doi:10.1038/s41598017-06057-7

Yu, C., Li, X., \& Zhang, W. (2015). Predicting adolescent problematic online game use from teacher autonomy support, basic psychological needs satisfaction, and school engagement: A 2-year longitudinal study. Cyberpsychology, Behavior, and Social Networking, 18(4), 228-233. doi:10.1089/ cyber.2014.0385 